\begin{tabular}{|c|c|c|}
\hline $\begin{array}{l}\text { PKS } \\
\text { PUBLIC } \\
\text { KNOWLEDGE } \\
\text { PROJECT }\end{array}$ & $\begin{array}{c}\text { REVISTA DE GEOGRAFIA } \\
\text { (RECIFE) } \\
\text { http://www.revista.ufpe.br/revistageografia }\end{array}$ & $\begin{array}{l}\text { OJS } \\
\frac{\text { OJEN }}{\text { OPENAL }} \\
\text { SYSTEMS }\end{array}$ \\
\hline
\end{tabular}

\title{
EVALUACIÓN DE SERVICIOS ECOSISTÉMICOS DE REGULACIÓN DE REMOCIÓN EN MASA Y EROSIÓN, EN LAS CUENCAS SALITRE Y TEUSACÁ, ÁREA RURAL DE BOGOTÁ, COLOMBIA
}

\author{
Julieth Monroy Hernández¹, Mónica Sandoval Ramos² \\ ${ }^{1}$ Jardín Botánico de Bogotá José Celestino Mutis. E-mail: jmonroy@jbb.gov.co \\ ${ }^{2}$ Jardín Botánico de Bogotá José CelestinoMutis. E-mail: monik.sandoval@gmail.com
}

Artigo recebido em $30 / 12 / 2017$ e aceito em 30/08/2018

\begin{abstract}
RESUMEN
Este artículo compila los resultados del proyecto de investigación "Evaluación de servicios ecosistémicos de regulación de remoción en masa y erosión, en las cuencas Salitre y Teusacá, área rural de Bogotá, Colombia", desarrollado por la línea de Biodiversidad y Servicios Ecosistémicos, de la Subdirección Científica del Jardín Botánico de Bogotá. El proyecto analiza la interacción de las coberturas vegetales con los procesos de erosión y remoción en masa, a partir del aporte en la prevención, dada la capacidad de interceptación de precipitación del follaje, tomando la lluvia como factor que incide en la aceleración de estos procesos. Se apoya en el análisis espacial y mapeo con un software SIG, lo cual proporciona la base de la observación relacional que difícilmente se lograría sin estas herramientas. Este trabajo busca ser un aporte a la evaluación de los servicios ecosistémicos de regulación, así como proveer información actualizada que sirva de base para estudios posteriores, relacionados con el concomimiento de las funcionalidades de la Estructura Ecológica Principal de Bogotá.

Palabras-clave: Servicios ecosistémicos, Remoción en masa, Erosión, Prevención, Coberturas, Interceptación.
\end{abstract}

\section{EVALUATION OF ECOSYSTEM SERVICES OF LANDSLIDE AND EROSION ADJUSTMENT, IN THE SALITRE AND TEUSACÁ BASINS, RURAL AREA OF BOGOTÁ, COLOMBIA.}

\begin{abstract}
This article compiles the results of the research "evaluation of ecosystem services of landslide and erosion adjustment, in the Salitre and Teusacá basins, rural area of Bogota, Colombia", by the Biodiversity and Ecosystem Services group of the Botanical Garden of Bogotá. This research analyzes the interaction of landcover with the processes of erosion and landslide, and their contribution to prevent this phenomenon, due to the foliage ability to intercept precipitation, taking the rain as a factor that affects the acceleration of these processes. It lies on spatial analysis and mapping with GIS software, which provides the basis for relational observation that would hardly be achieved without these tools. This work seeks to be a methodological contribution for the evaluation of regulatory ecosystem services and provide updated information that will be used in future studies related to the functionalities of the Main Ecological Structure of Bogotá.
\end{abstract}

Keywords: Ecosystem services, Mass removal, Erosion, Prevention, Coverage, Interception. 


\section{INTRODUCCIÓN}

El distrito de Bogotá tiene al interior de su territorio zonas montañosas, caracterizadas por sus altas pendientes, que las hacen susceptibles a eventos de remoción en masa y erosión. Gran parte de estas zonas están amparadas por figuras de protección y conservación, que han permitido el desarrollo de vegetación de diferente tipo que, sin embargo, muestran evidentes contrastes con otros usos propios de las urbes y aquellos relacionados a las zonas periurbanas, como son los usos agropecuarios. Con el fin de evaluar las áreas que prestan servicios ecosistémicos de regulación de remoción en masa y erosión, la investigación que se presenta a continuación, realiza una zonificación de aquellos procesos activos y no activos, relacionando estos con los servicios de prevención que pueden generar las coberturas, a partir de su capacidad de interceptación de lluvia, la cual es un agente que potencializa la ocurrencia de dichos eventos. Se centra en el área rural de Bogotá en la zona alta de las cuencas de los ríos Salitre y Teusacá (Figura 1).

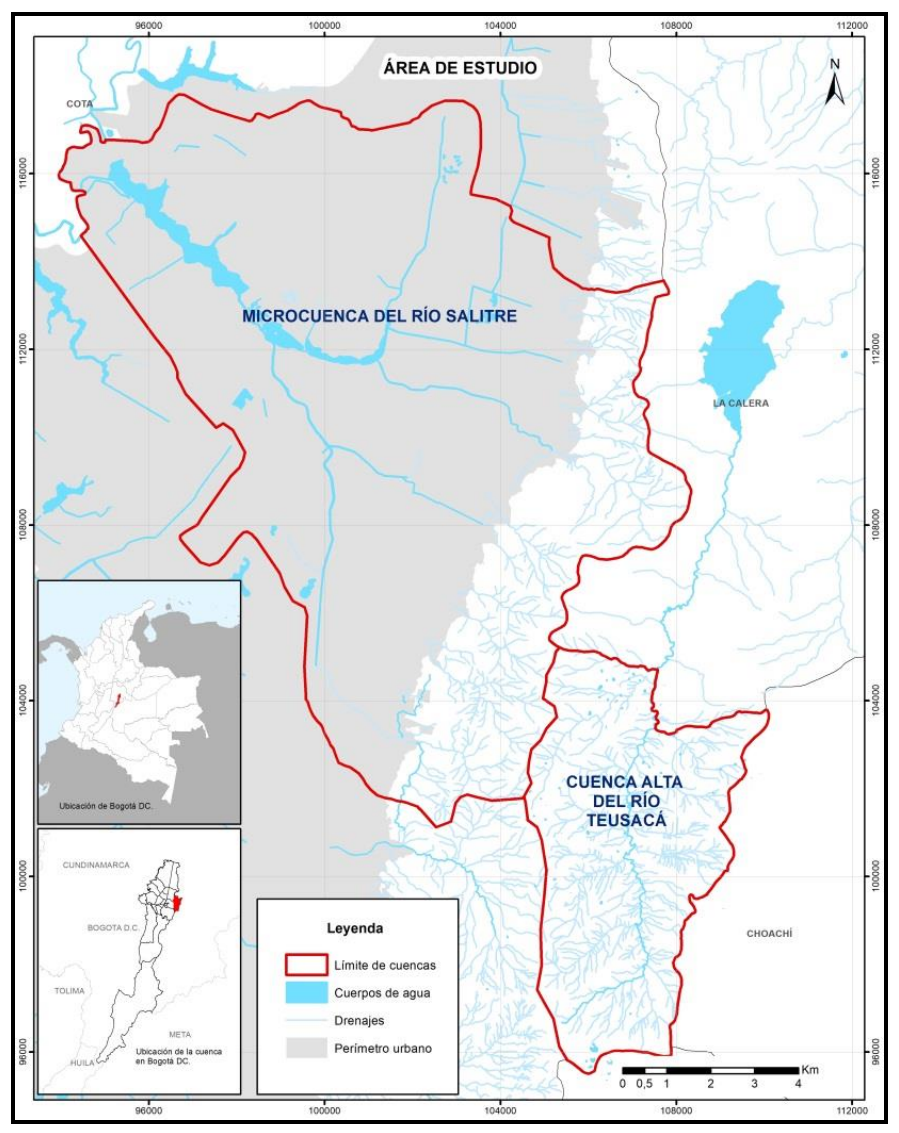

Figura 1. Mapa de ubicación del área de estudio. Elaboración propia. Fuente de cartografía base: IDECA 
Para realizar los análisis planteados, fue necesaria la actualización y el ajuste de información cartográfica, ya que Bogotá no cuenta con suficientes datos a una misma escala, que permitan generar observaciones a fondo sobre fenómenos como la erosión o la remoción en masa, por lo cual la escala a la que logran llegar los resultados es 1:25.000. De esta manera, el estudio hace un acercamiento metodológico que busca aportar a la evaluación de servicios ecosistémicos de regulación, en la medida en que une elementos de diferentes disciplinas como la hidrología, la agrología y la biología, interactuando con procedimientos de los sistemas de información geográfica.

Este estudio es realizado por la línea de investigación en Biodiversidad y Servicios Ecosistémicos, de la Subdirección Científica del Jardín Botánico de Bogotá José Celestino Mutis, en el marco del proyecto de inversión 1121 (2016 al 2020), el cual se plantea como uno de sus objetivos, el caracterizar y valorar ecológicamente los elementos de la Estructura Ecológica Principal del Distrito Capital.

\section{SERVICIOS ECOSISTÉMICOS PARA LA PREVENCIÓN DE LA REMOCIÓN EN MASA Y LA EROSIÓN}

Los servicios ecosistémicos son definidos por la Evaluación de Ecosistemas del Milenio (2003), como aquellos beneficios obtenidos de los ecosistemas a través de diferentes procesos que aseguran su sostenibilidad; esto incluye servicios de provisión como alimento y agua; servicios de regulación como regulación de inundaciones, sequias y degradación de tierras; servicios de soporte como la formación de suelo y el ciclaje de nutrientes; y servicios culturales como los de recreación, espirituales, religiosos y otros beneficios no materiales.

Esta perspectiva, aunque utilitaria y antropocéntrica, permite hacer visibles los beneficios que genera la naturaleza, desde el punto de vista de la planeación y la gestión pública. Es así como a través de la caracterización y valoración de los ecosistemas como prestadores de servicios, la importancia de conservar la naturaleza deja de ser un enunciado general dentro de las normas y se empieza a abrir camino en la gestión y planificación territorial, siempre con miras al ámbito productivo (Ministerio de Ambiente y Desarrollo Sostenible, 2012)

En el contexto de la presente investigación se resaltan los servicios ecosistémicos de regulación, los cuales son definidos como los beneficios obtenidos gracias a la tendencia de 
los ecosistemas a mantener sus procesos y funciones naturales; como el mantenimiento de la calidad del aire, regulación del clima, regulación hídrica, control de la erosión, purificación del agua y tratamiento de desechos, regulación de enfermedades humanas, control biológico, polinización y protección ante tormentas. Este tipo de servicios son prestados de forma directa por los ecosistemas, sin intermediación del mercado o transformaciones de infraestructura, como es común para otros servicios ecosistémicos como los de provisión (Millennium Ecosystem Assessment, 2003).

Los fenómenos de remoción en masa y erosión se pueden considerar un servicio de regulación, ya que se presentan como manifestaciones naturales de los ciclos geomorfológicos, que buscan mantener el equilibrio dinámico entre las fuerzas constructoras del relieve -como los esfuerzos internos que generan el crecimiento de las cordilleras- y las fuerzas destructivas, que configuran los modelados y van reduciendo constantemente el tamaño de las montañas (Flórez 2003). La interacción de estos procesos con la vegetación, en especial con pendientes altas, hace que la probabilidad de ocurrencia de un evento de remoción en masa o erosión se reduzca, o por lo menos mitigue sus efectos, por lo que también se convierte en un elemento importante dentro del sistema de regulación. En el caso de la erosión, la vegetación amortigua el golpe de la precipitación sobre el suelo y ayuda a reducir el volumen de agua que se infiltra, disminuyendo el lavado de partículas (León Peláez 2001), y en la medida en que las coberturas vegetales sean más densas y robustas, presenten varios estratos y una protección directa del suelo -bien sea con hojarasca, musgos o herbáceas-, tienen un mayor potencial de ayudar en la prevención de movimientos en masa y erosión (Ramos, Trujillo-Vela y Prada 2015).

En el caso de los cerros bogotanos, diversos estudios han evidenciado que, gran parte de las zonas con pendientes superiores al $15 \%$ se encuentran cubiertas por pastizales o por viviendas, que no siempre tienen la infraestructura adecuada para controlar la estabilidad del terreno; esta situación se acentúa por el avance de la deforestación presente aun en los bosques y páramos del Distrito, los cuales han sido declarados como áreas protegidas destinadas para la conservación (CAR 2006). 


\section{METOdOLOGÍA PARA LA ZONIFICACIÓN DE COBERTURAS, PROCESOS EROSIVOS Y DE REMOCIÓN EN MASA}

El proyecto se dividió en tres fases, en las cuales se utiliza como herramienta fundamental un software de Sistemas de Información Geográfica (SIG), en el que se llevan a cabo varios procesos de análisis espacial. En la primera fase se identificaron procesos erosivos, procesos de remoción en masa, coberturas y pendientes, este último elemento se integra de forma trasversal en los diferentes procesos de análisis.

Para realizar la zonificación de los procesos erosivos de ambas cuencas, se evaluó la erosión potencial y la erosión real. Para hallar la erosión potencial se utilizó la ecuación universal de pérdida del suelo - USLE (Universal Soil Loss Equation), la cual es un modelo que tiene en cuenta la energía de la lluvia como agente erosivo y las características del terreno sujeto de la erosión, como la longitud y grado de inclinación de la pendiente, las características físicas del suelo, cobertura y uso y las prácticas de manejo como la mecanización o conservación (Renard, Foster, Weesies, McCool, \& Yoder, 1997).

Sin embargo, para este estudio no se toman los datos de uso del suelo (Factor C), asumiendo que la cobertura es homogénea en la determinación de la erosión potencial, de esta forma se puede comparar la erosión potencial con la real sin tener en cuenta el aporte de la cobertura vegetal, para deducir preliminarmente dónde se podrían estar prestando el servicio ecosistémico de prevención de la erosión. También se descarta el factor de práctica mecánica de apoyo o prácticas de conservación (Factor P), ya que ambas cuencas se caracteriza por pendientes que no permiten la mecanización, por lo que se asume que las prácticas de manejo del suelo son similares para toda el área de estudio. De esta forma, los factores que componen la USLE, se estiman tomando como base el estudio general de suelos y zonificación de tierras de Cundinamarca (IGAC, 2000), del cual se realizó una revisión de las fases con la ayuda de un Modelo Digital de Elevación (DEM) con precisión de 12,5 metros.

Una de las variables más importantes, es la erosionabilidad o Factor K del suelo, que se define como la resistencia de un suelo a desintegrarse por la acción del agua. Este análisis se realiza por medio de la ecuación de Wischmeier y Smith, referenciada por Markov y Nedkov (2016), la cual se basa en la granulometría (porcentaje de arena, arcilla y limo), el porcentaje de 
materia orgánica, la estructura y la permeabilidad del suelo. Luego se calculó la erosión real, siguiendo la metodología del "Protocolo para la identificación y evaluación de la degradación de suelos por erosión" (IDEAM, MADS, UDCA, 2015), a partir del análisis de la imagen de satélite SPOT 2016 para Bogotá, el Modelo Digital de Elevación (DEM), imágenes de Digital Globe disponibles en Google Earth y la verificación en campo.

Esta zonificación evidencia que, aunque potencialmente se calcula un alto porcentaje de erosión, debido a las formas y longitud de la pendiente y el factor de erosionabilidad del suelo, solo en pocos casos se encuentran sectores con procesos erosivos activos, pues estos se dan aproximadamente en un $2 \%$ del territorio analizado. De esta forma, se podría inferir que existen factores que aportan a los servicios ecosistémicos de prevención de la erosión, allí donde la amenaza es alta y no se encuentran evidencias de este fenómeno.

Para determinar la amenaza de remoción en masa en las cuencas Salitre y Teusacá se tomó la zonificación vigente en el Plan de Ordenamiento Territorial (POT) de Bogotá (Decreto 190 de 2004). Esta información alcanza a cubrir únicamente el área que presenta algún tipo de amenaza de movimientos en masa dentro de la cuenca Salitre, por lo que respecto al área de la cuenca del río Teusacá, se calculan áreas potenciales a ser susceptibles a eventos de remoción en masa, a partir de información secundaria que aporta el Plan de Manejo de la Reserva Forestal Bosque Oriental de Bogotá (CAR 2006), siguiendo la Guía Metodológica para estudios de amenaza, vulnerabilidad y riesgo por movimientos en masa del Servicio Geológico Colombiano (2015). Igualmente se realiza la identificación de la remoción real, para cada una de las cuencas, a partir del análisis de una imagen Spot de 2016, los puntos del inventario de eventos de remoción en masa del Instituto Distrital de Gestión de Riesgos y Cambio Climático - IDIGER y verificación en campo. Al igual que la erosión, en el caso de los procesos de remoción en masa, el comportamiento es similar, encontrándose procesos activos cercanos al área urbana, relacionados a la inestabilidad de laderas en áreas intervenidas.

Se infiere que, en ambos casos aquellas zonas que no se encuentran activas y que poseen características de amenaza media y alta a los movimientos en masa y la erosión, en condiciones de pendiente moderada a alta (mayor al 25\%), y alto factor de erodabilidad del suelo, prestan servicios ecosistémicos de prevención. 
Posteriormente se realiza el análisis y actualización de coberturas a escala 1:25.000, tomando como base el estudio de coberturas realizado por la CAR en 2006 y el estudio realizado por el IDEAM (2003) para el periodo 2000 - 2002. Siguiendo la metodología Corine Land Cover, adaptada para Colombia, se obtiene que las coberturas que presentan una mayor extensión en ambas cuencas son: herbazales, plantaciones, pastos y vegetación secundaria, predominando la cobertura de pastos, intercalados con cultivos en la cuenca del río Teusacá, y una gran extensión de eucaliptos, plantaciones de pinos y vegetación secundaria en la microcuenca Salitre.

\section{APORTE DE LAS COBERTURAS VEGETALES EN LA PREVENCIÓN DE LA EROSIÓN Y LA REMOCIÓN EN MASA}

En esta segunda fase, se caracterizó la relación de los tipos de coberturas y el suelo, a partir de la interceptación de la precipitación, basándose en la hipótesis en que la vegetación reduce el proceso físico de fricción de la lluvia sobre el suelo, lo cual causa pérdida de partículas superficiales, proceso que es acelerado por la pendiente. La información de coberturas se intersecta con las zonificaciones de procesos de remoción y erosión, analizando solo aquellas zonas donde, a pesar de presentar pendientes mayores al $25 \%$ y suelos con alto factor de erodabilidad, no se encuentran activas. De este resultado se toman aquellas coberturas de mayor extensión, que son comunes a las áreas que potencialmente prestan aportes a la prevención: eucaliptos, pinos, herbazales y vegetación secundaria. En estas se realiza la medición de los porcentajes de interceptación del follaje, ubicando parcelas en el predio Horizontes (costado occidental de los cerros de Bogotá, parte alta de la cuenca del río Salitre, el cual es administrado por la Fundación Cerros de Bogotá), donde se realizaron observaciones durante 9 semanas, en los meses de alta precipitación, entre octubre y noviembre de 2017.

Para esta medición se retoma la metodología de Mateos-Rodríguez \& Schnabel (1998), en la cual se disponen una serie de recipientes bajo la vegetación a estudiar, para recolectar la lluvia que cae al suelo luego de la interceptación; igualmente se dispone un recipiente descubierto para medir la precipitación neta del sector y poder comparar la cantidad de lluvia interceptada, ya que debido a la variabilidad de la lluvia por la incidencia del contexto de montaña, la 
precipitación difiere de las mediciones de las estaciones meteorológicas con las que cuenta el Distrito de Bogotá (la más cercana está ubicada a $6 \mathrm{~km}$ del punto de medición).

De acuerdo con Méndez Monroy (2013), se puede tener un valor experimental de la interceptación, calculando la diferencia entre la cantidad de precipitación incidente, que para este caso se denomina precipitación neta $(\mathrm{Pn})$, y la cantidad del flujo libre de translocación $(\mathrm{TH})$ y goteo $(\mathrm{G})$, que en este caso es precipitación en suelo (Ps). En esta operación se presume que el follaje antes del evento de precipitación se encuentra seco, y que el agua almacenada durante el periodo de medición del evento se evapora totalmente desde el follaje. Por lo tanto, el cálculo de porcentaje de interceptación de cada cobertura seleccionada será la proporción entre Pn y Pt.

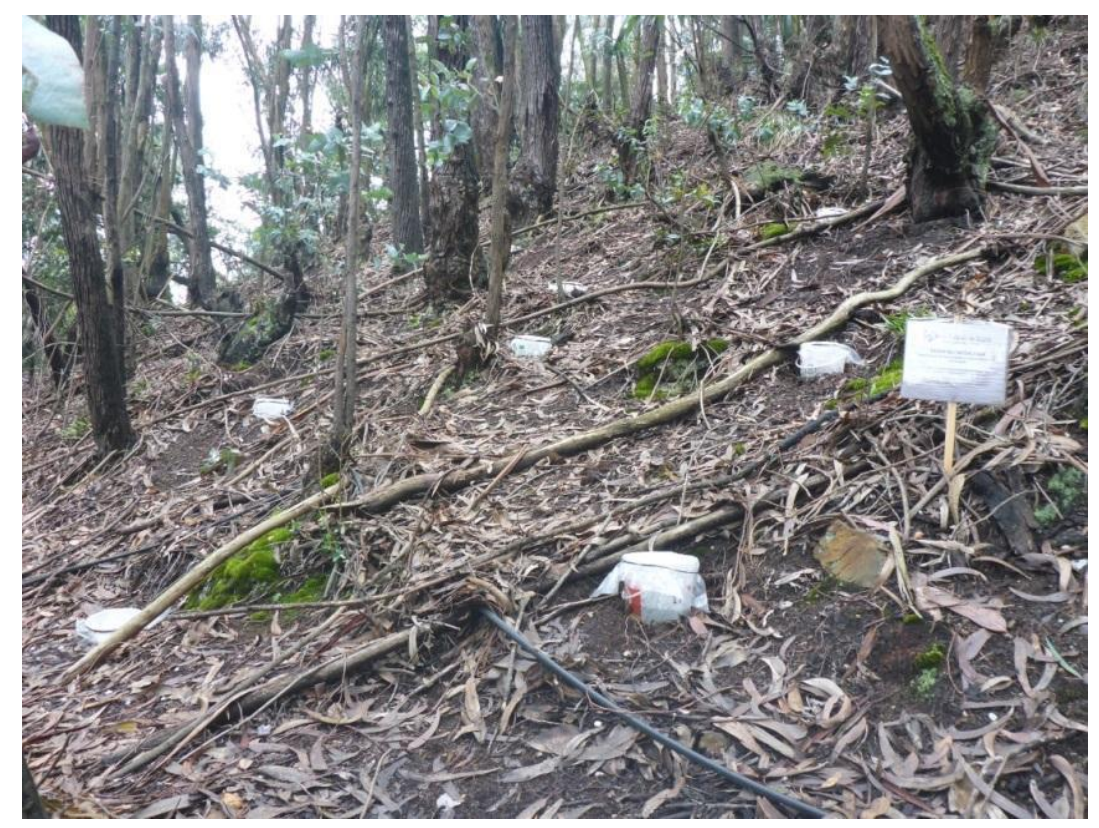

Figura 2. Instalación de pluviómetros simples en cobertura de Eucalipto.

A partir de las mediciones, se realizó un análisis comparativo del comportamiento de las coberturas frente a la interceptación de lluvia en su follaje, considerando tres eventos: precipitación mínima, precipitación máxima y eventos atípicos. Las precipitaciones mínimas se presentaron en las semanas de 9 al 15 de octubre, con $3 \mathrm{~mm}$ y del 16 al 22 de octubre con $1 \mathrm{~mm}$. Para tener una mejor comparación de los datos se toma la primera semana mencionada, en la cual se encuentra que la cobertura que menor aporte tiene en la interceptación de la precipitación es el eucalipto. 


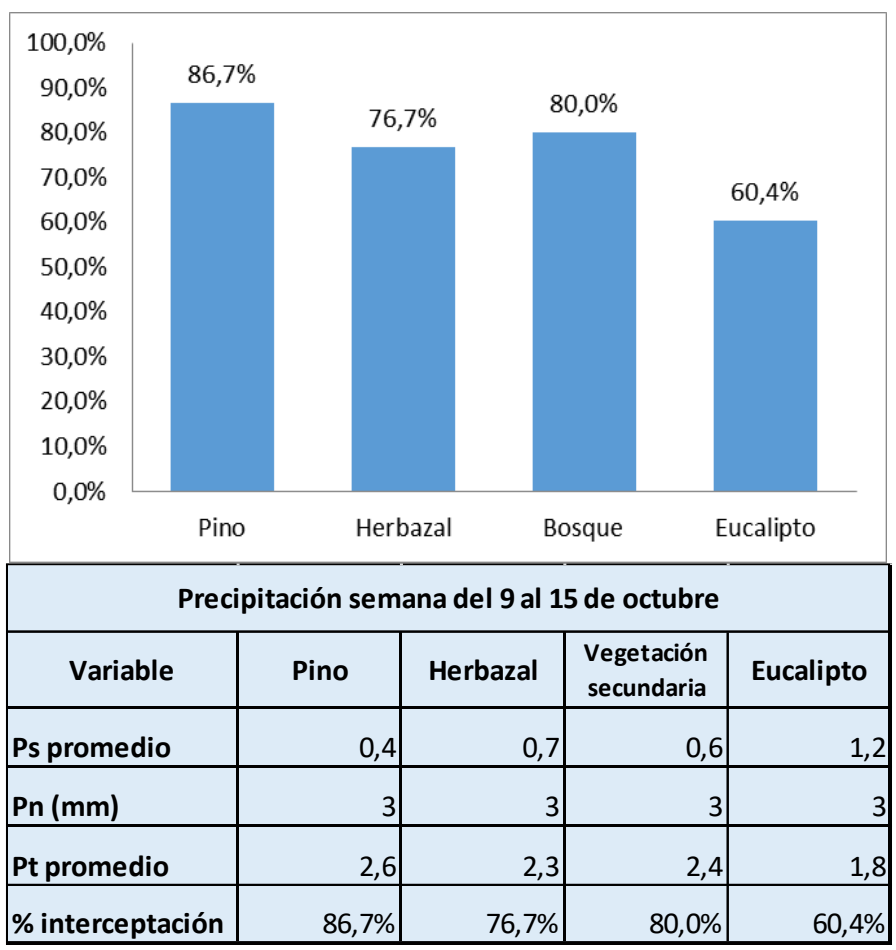

Figura 3. Relación de porcentajes de interceptación por coberturas en periodos de precipitación mínima

En general todas las coberturas mantienen un alto porcentaje de interceptación, esto puede indicar que, en condiciones de baja precipitación, cada cobertura aprovecha gran parte del recurso agua a través de su follaje, por lo que la precipitación en suelo es baja.

La precipitación máxima (exceptuando el evento atípico), se presentó en la semana del 23 al 29 de octubre con $43 \mathrm{~mm}$ de lluvia. Se observa que el aporte a la interceptación de la cobertura de eucalipto es muy bajo, permitiendo que el promedio de la Ps aumente considerablemente. Por su parte las coberturas de herbazal y vegetación secundaria mantienen un promedio de interceptación similar, mientras que la cobertura de pino es la que mayor aporte genera. 


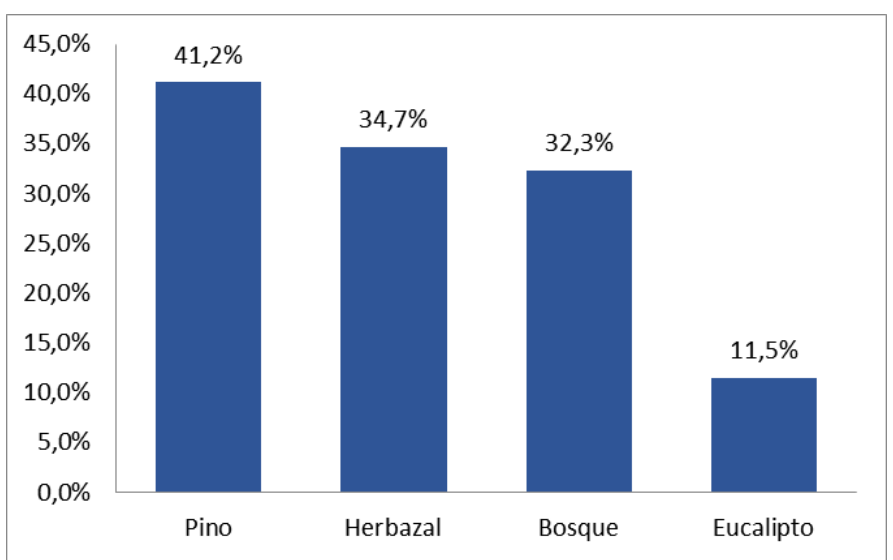

\begin{tabular}{|l|r|r|r|r|}
\hline \multicolumn{5}{|c|}{ Precipitación semana del 23 al 29 de octubre } \\
\hline \multicolumn{1}{|c|}{ Variable } & \multicolumn{1}{|c|}{ Pino } & Herbazal & $\begin{array}{r}\text { Vegetación } \\
\text { secundaria }\end{array}$ & Eucalipto \\
\hline Ps promedio & 25,3 & 28,1 & 29,1 & 38,06 \\
\hline Pn (mm) & 43 & 43 & 43 & 43 \\
\hline Pt promedio & 17,7 & 14,9 & 13,9 & 4,94 \\
\hline$\%$ interceptación & $41,2 \%$ & $34,7 \%$ & $32,3 \%$ & $11,5 \%$ \\
\hline
\end{tabular}

Figura 4. Relación de porcentajes de interceptación por coberturas en periodos de precipitación máxima

Se observa que a medida que la precipitación aumenta, supera la capacidad del follaje de las coberturas, en especial en eucaliptos, permitiendo que más lluvia logre entrar en contacto con el suelo. Esto sumado a la pendiente y un alto factor de erodabilidad del suelo, puede aumentar la probabilidad de ocurrencia de eventos de erosión, y dependiendo de la estructura física del suelo y de la litología del área, también pueden surgir eventos de remoción en masa.

El comportamiento de las coberturas frente a la precipitación en un evento atípico, mantiene la relación registrada en alta precipitación, sin embargo, su porcentaje de interceptación no alcanza a superar el 40\%. El eucalipto continúa siendo la cobertura que menor aporta, y por lo tanto permite que la Ps aumente, influyendo así en la incidencia sobre los fenómenos analizados. Las coberturas de pino, herbazales y la vegetación secundaria no varían considerablemente su aporte a la interceptación, por el contrario, se mantienen. 


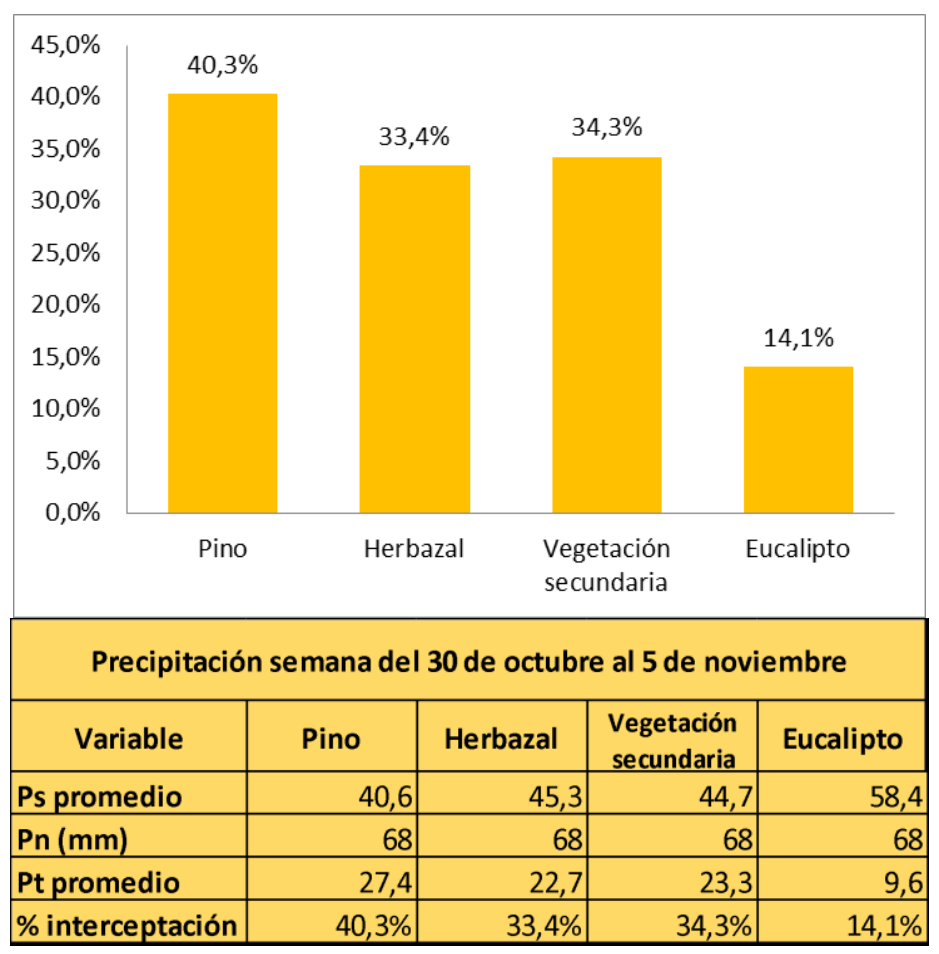

Figura 5. Relación de porcentajes de interceptación por coberturas en un evento atípico (granizada del 2 de noviembre de 2017)

\section{EVALUACIÓN DE SERVICIOS ECOSISTÉMICOS DE PREVENCIÓN DE REMOCIÓN EN MASA Y EROSIÓN}

Con base en estos resultados obtenidos, se realiza la valoración de las coberturas que mayor aportan a la prevención de procesos de remoción en masa y erosión, a partir de una escala cualitativa que califica el aporte como bajo, medio o alto, para cada cobertura observada, extrapolando la valoración de acuerdo a su asociación con la unidad de cobertura, según la clasificación de la metodología Corine Land Cover definida por el IDEAM (2010). De esta forma las plantaciones se califican dentro de un nivel medio y bajo. En la cobertura de Eucalipto su nivel de aporte se califica como bajo, ya que en las áreas donde se encuentran estas plantaciones, el suelo está casi desprovisto de otro tipo de vegetación, por lo que la incidencia de la precipitación sobre el factor de erodabilidad del suelo es alta.

En el caso de los pinos, su nivel de aporte se califica como medio, ya que a pesar de ser la cobertura que mayor precipitación intercepta, y a que sus acículas que caen sobre el suelo se 
convierten en una capa que recubre la superficie, evitando así la acción del agua como elemento erosivo, su aporte frente a la prevención de remoción y erosión con respecto a la acción de sus raíces, es un factor que debe medirse por separado, ya que, como menciona Guerrero Campo (1998) es un tipo de cobertura que influye en la degradación del suelo.

Los otros tipos de plantaciones, se califican en un nivel bajo. Este tipo de coberturas de acción antrópica, por lo general se componen de árboles de la misma edad y altura, distribuidos uniformemente sobre el terreno, sin asociación con otros tipos de vegetación, permitiendo así que la acción de la Ps sea mayor, y por lo tanto aumenten las condiciones de amenaza frente a procesos de erosión y remoción en masa en pendientes altas.

Las coberturas herbáceas y/o arbustivas, comprenden la unidad donde se encuentran herbazales de diferente tipo, arbustales y vegetación secundaria. Se califican con un aporte alto ya que, de acuerdo a las mediciones obtenidas, mantienen una relación más constante frente a la interceptación, en especial en los eventos de precipitación máxima.

Para el caso de los pastos, se evalúan con base en información secundaria según Méndez Monroy (2013) y Ataroff y Rada (2000). Los pastos por ser una cobertura de acción antrópica, discontinua e intercalada con sectores arbolados, enmalezados o áreas de cultivo, su aporte es bajo, ya que la interceptación es menor a la que se presenta en bosques y áreas de vegetación herbácea (Méndez Monroy 2013), y en un contexto de montaña andina, esta cobertura reduce las entradas de precipitación y la intercepción de lluvia del área Ataroff y Rada (2000).

Los resultados indican que, a pesar que las áreas encontradas en la zonificación de procesos erosivos y de remoción en masa no activas, presumiblemente se encuentran prestando un servicio ecosistémico de prevención, al encontrarse en interacción constante con diferentes tipos de coberturas, el aporte al SE es dinámico y puede cambiar con el tiempo. Las áreas que mayor aporte generan a la prevención de los procesos analizados se ven reducidas en función de la extensión de las coberturas menos intervenidas, como lo son los herbazales y la vegetación secundaria, mientras que las coberturas que han tenido mayor influencia de la acción antrópica, como las plantaciones y pastos, tienen un área mayor que proporciona un aporte menor al sostenimiento del SE de prevención que se podría estar dando. 


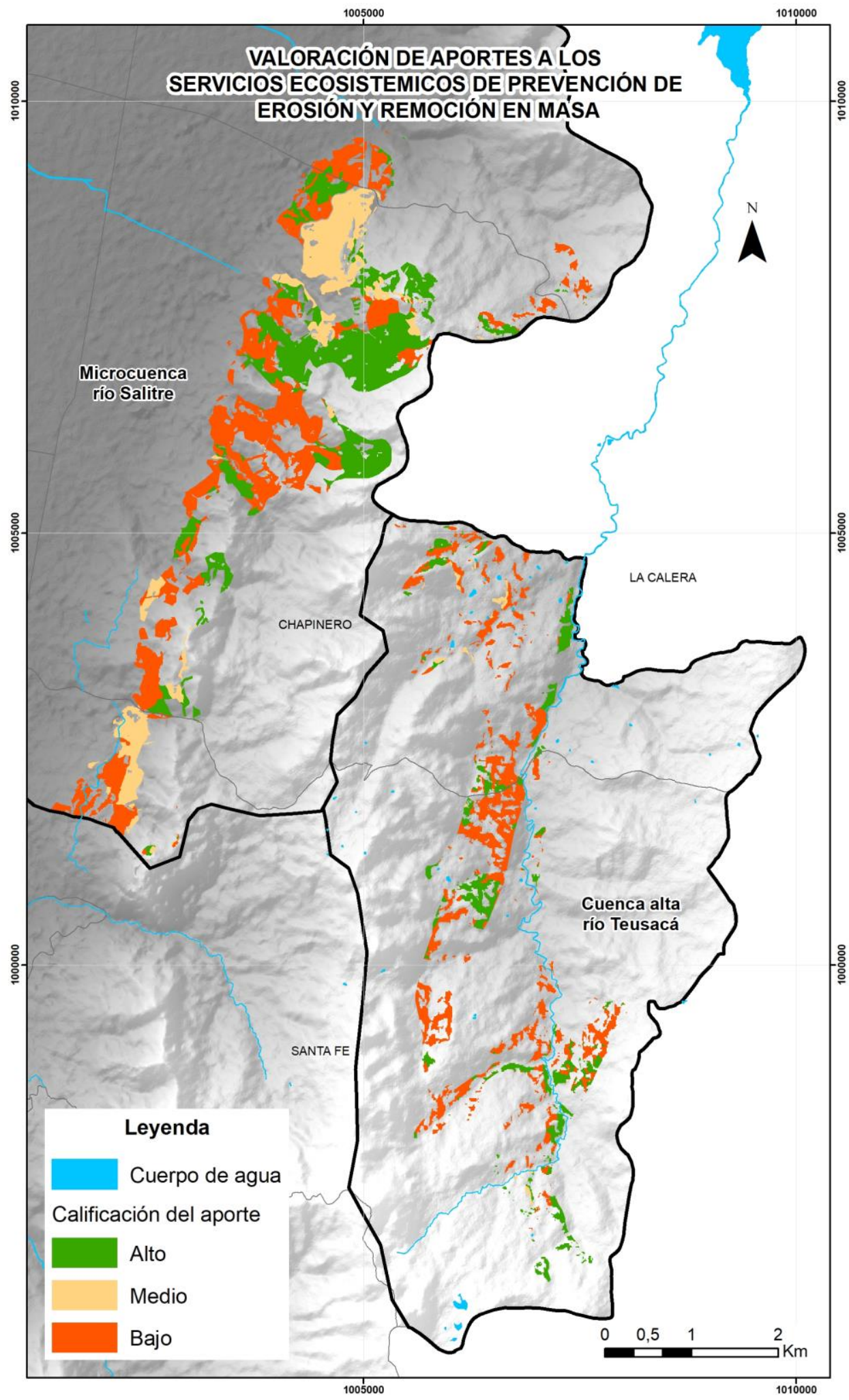

Figura 6. Mapa de valoración de aportes al SE de prevención de procesos de erosión y remoción en masa. 


\section{CONCLUSIONES}

En cuanto a la zonificación de procesos de remoción en masa y erosión en las cuencas Salitre y Teusacá, los diferentes procedimientos desarrollados requirieron del uso de información secundaria, que para el caso del río Teusacá se encuentra incompleta. Por tal razón, los resultados presentados para la cuenca alta del río Teusacá son indicativos y se sugiere un estudio de base para complementar la información aquí utilizada.

La zonificación de procesos erosivos obtenida, permite observar que, a pesar de tener amplias áreas con tendencia a la erosión, ambas cuencas presentan poca frecuencia de eventos, limitándose por ejemplo los de erosión a zonas que tienen algún uso de tipo antrópico. En el caso de los procesos de remoción en masa, el comportamiento es similar, encontrándose procesos activos cercanos al área urbana, relacionados a la inestabilidad de laderas en áreas intervenidas. Se infiere que, aquellas zonas que no se encuentran activas y que poseen características de amenaza media y alta a los movimientos en masa y la erosión, en condiciones de alta pendiente y alto factor de erodabilidad del suelo, están prestando servicios ecosistémicos de prevención.

De esta forma, a partir de la identificación de áreas no activas con alto grado de vulnerabilidad física a la erosión y la remoción en masa, se logran identificar coberturas para la evaluación de su aporte al SE de prevención a partir de la medición de porcentajes de interceptación de precipitación por medio de su follaje. Este análisis se basa en que, las coberturas actúan como un reductor de las lluvias y su influencia en la distribución espacial de la infiltración, ayuda a la disminución de la escorrentía superficial y por lo tanto a la pérdida de suelo y/o saturación de este, eventos que aumentarían la probabilidad de movimientos en masa condicionados por la pendiente.

La cobertura de eucalipto fue la de menor porcentaje de interceptación, con un promedio de 11,5\% de interceptación en eventos de alta precipitación, es decir entre un 20\% a 30\% menos que las otras coberturas analizadas. Como se pudo apreciar en campo, el suelo se encuentra casi desprovisto de otro tipo de vegetación, por lo que la incidencia de la precipitación sobre el factor de erodabilidad del suelo es alta. Sin embargo, los restos de hojas de eucalipto podrían proveer un elemento de favorabilidad en la disminución de la fricción que puede 
ocasionar la lluvia sobre el suelo. En el caso de los pinos las acículas que caen sobre el suelo se convierten en una capa que cubre casi totalmente la superficie, evitando así la acción del agua como elemento erosivo. Se considera que las coberturas de menor intervención son las que más aportan a la interceptación de lluvia, pues mantienen un porcentaje poco variable, como se mencionó anteriormente, en especial en eventos de alta precipitación, manteniendo un promedio de interceptación entre el 30\% y 35\%.

En las áreas seleccionadas para observación, según la zonificación de coberturas que aportan a la prevención, aún no es evidente casos de erosión o remoción en masa de gran escala, esto se debe posiblemente a que se mantiene una cobertura variada y continúa con poca intervención. Algunos senderos no artificializados presentan erosión inicipiente debido al paso de caminantes, mientras sobre el borde construido se pueden identificar casos de movimientos en masa, como a lo largo de la avenida circunvalar, intervención que ha afectado la estabilidad de las laderas.

Cabe desatacar que, la forma en que las coberturas vegetales pueden aportar en la prevención de procesos de erosión y remoción en masa es compleja, pues no solo se define a partir de su potencial como interceptoras de lluvia, sino también por la acción de las raíces y el aporte de la vegetación a la estructura del suelo. Se propone este tipo de análisis como una segunda fase del proyecto, lo cual ayudaría a complementar la información recolectada, con el fin de comprender mejor la relación entre las coberturas y el suelo.

Dado que los procesos de erosión y remoción son dinámicos, para llegar a una escala más detallada, se requiere de información base a mayor escala y realizar una medición en un periodo de tiempo más largo. Por lo tanto, se considera necesario, que las instancias oficiales amplíen la información base para el desarrollo de investigaciones que aporten al conocimiento de los sistemas naturales que rodean el Distrito. Esta actualización de información y ampliación de la escala de trabajo, permitirá tener resultados más fiables que aporten a una mejor planificación y gestión del territorio. 


\section{REFERENCIAS}

ALCALDÍA MAYOR DE BOGOTÁ. Mapa de Amenaza por Remoción en Masa. Decreto Distrital 190 de junio 22 de 2004 "Por medio del cual se compilan las disposiciones contenidas en los Decretos Distritales 619 de 2000 y 469 de 2003", Plan de Ordenamiento Territorial del Distrito Capital. Bogotá. 2004

ATAROFF, M., \& RADA, F. Deforestation Impact on Water Dynamics in a Venezuelan Andean Cloud Forest. Ambio Vol. 29 N 1. Royal Academy of Sciences. 2000.

CAR. Plan de manejo de la Reserva Forestal Protectora Bosque Oriental de Bogotá. Documento principal. Corporación Autónoma Regional de Cundinamarca, CAR. Bogotá: 2006

FLÓREZ, ANTONIO. Colombia evolución de sus relieves y modelados. Red de Estudios de Espacio y Territorio - RET. Universidad Nacional de Colombia. Bogotá, Colombia. 2003 GUERRERO CAMPO, J. Respuesta de la vegetación y de la morfología de las plantas a la erosión del suelo. Serie investigación. Consejo de Protección de la Naturaleza de Aragón. Zaragoza. 1998

IDEAM. Coberturas de la Tierra Periodo I 2000 - 2002. Metodología Corine Land Cover adaptada para Colombia. Instituto de Hidrología, Meteorología y Estudios Ambientales. Bogotá, Colombia. 2003.

IDEAM. Leyenda Nacional de Coberturas de la Tierra. Metodología CORINE Land Cover adaptada para Colombia escala 1:100.000. Instituto de Hidrología, Meteorología y Estudios Ambientales. Bogotá DC: 72p. 2010

IGAC. Suelos de Colombia: origen, evolución, clasificación, distribución y uso. Ministerio de Hacienda y Crédito Público. Bogotá: 1995.

IDEAM, MADS, UDCA. Protocolo para la identificación y evaluación de la degradación de suelos por erosión. Instituto de Hidrología, Meteorología y Estudios Ambientales. Bogotá. 2015.

IGAC. Estudio general de suelos y zonificación de tierras del departamento de Cundinamarca. Bogotá. Instituto Geográfico Agustín Codazzi. Bogotá. 2000.

LEÓN PELÁEZ, J. Estudio y control de la erosión hídrica. Medellín: Universidad Nacional de Colombia. Sede Medellín. 2001.

MARKOV, B., \& NEDKOV, S. Mapping of erosion regulation ecosystem services. Proceedings, 6th International Conference on Cartography and GIS. 2016. 
MATEOS-RODRÍGUEZ, A., \& SCHNABEL, S. Medición de la interceptación de las precipitaciones por la encina (Quercus Rotundifolia Lam.): Metodología e instrumentación. Norba (10), 95-112. 1998.

MÉNDEZ MONROY, JAVIER FERNANDO. Metodología para la medición de variables hidrometeorológicas que faciliten la implementación de modelos dinámicos de interceptación de lluvia en el contexto Colombiano. Tesis de Maestría, Universidad Nacional de Colombia, Facultad de Ingeniería, grupo de investigación en Ingeniería de Recursos Hídricos. Bogotá. 2013.

MILLENNIUM ECOSYSTEM ASSESSMENT. Ecosystems and human well-being: a framework for assessment. Island Press. Washington DC: 2003.

MINISTERIO DE AMBIENTE Y DESARROLLO SOSTENIBLE. Política Nacional para la Gestión Integral de la Biodiversidad y sus Servicios Ecosistémicos (PNGIBSE). Bogotá. 2012.

RAMOS C., A., TRUJILlO-VELA, M., \& PRADA S., L. Análisis descriptivos de procesos de remoción en masa en Bogotá. Obras y Proyectos 18, 63-75. 2015.

RENARD, K., FOSTER, G., WEESIES, G., MCCOOL, D., \& YODER, D. Predicting soil erosion by water: a guide to conservation planning with the revised Universal Soil Loss Equation (RUSLE). USDA Agricultural Hankbook (703). 1997.

SERVICIO GEOLÓGICO COLOMBIANO. Guía Metodológica para estudio de amenaza, vulnerabilidad y riesgo por movimientos en masa. Imprenta Nacional. Bogotá, Colombia: 2015. 